\title{
Reoperation Rates Following Open-Door Cervical Laminoplasty
}

\author{
JOHN A. RODRIGUEZ-FEO, MD, ${ }^{1}$ DANIEL LEAS, MD,${ }^{1}$ SUSAN M. ODUM, PhD ${ }^{2}$ MARC ANGERAME, \\ MD,${ }^{1}$ MARK KURD, MD,${ }^{3}$ BRUCE V. DARDEN, MD,${ }^{4}$ R. ALDEN MILAM, MD ${ }^{4}$ \\ ${ }^{I}$ Department of Orthopaedic Surgery, Carolinas Medical Center, Charlotte, North Carolina, ${ }^{2}$ OrthoCarolina Research Institute Inc., Charlotte, North Carolina, \\ ${ }^{3}$ Thomas Jefferson University \& Rothman Institute, Philadelphia, Pennsylvania, ${ }^{4}$ OrthoCarolina Spine Center, Charlotte, North Carolina
}

\begin{abstract}
Background: Laminoplasty is a safe and effective procedure for multilevel degenerative cervical stenosis causing myelopathy, that allows for motion preservation. The purpose of this study was to determine the reoperation rate and associated risk factors after cervical laminoplasty.

Methods: We present a retrospective consecutive series of patients who underwent a laminoplasty procedure between January 1, 2005, and October 31, 2012, and had a minimum 2-year follow-up. Demographic data were collected and operative records were reviewed to determine the cervical levels involved in the laminoplasty procedure, any concomitant procedures, and estimated blood loss. Clinical records were reviewed and telephone interviews were conducted on those patients with less than 2-year in-person follow-up. Patients requiring reoperations and the reason for the reoperations were determined, as well as the incidence of postoperative C5 palsy.

Results: A total of 222 of 266 patients (83\%) with a minimum 2-year follow-up had an average follow-up of 4.97 years. Overall, 26 patients underwent 30 reoperations (13.5\%). A total of 15 patients underwent 16 reoperations $(7.2 \%)$ in the acute postoperative period for infection requiring at least 1 irrigation and debridement, hardware-related issues, or posterior cervical wound issues. A total of 13 patients had 14 reoperations $(6.3 \%)$ outside of the acute postoperative period for the development of a new radiculopathy, recurrent myelopathy, neurologic symptoms with a kyphotic deformity, or a posttraumatic focal kyphotic deformity. Patients who had a concomitant laminectomy demonstrated a significantly $(P=.03)$ higher reoperation rate than those who did not. There were no other statistically significant associations. The C5 palsy rate was $8.1 \%$ (18 of 222).

Conclusions: Although the preserved motion following laminoplasty may allow for the development of new neurologic symptoms, the reoperation rate continues to compare favorably with laminectomy and fusion and remains a reasonable option for the surgical management of multilevel cervical stenosis causing myelopathy.
\end{abstract}

Level of Evidence: 4.

Cervical Spine

Keywords: laminoplasty, cervical spondylotic myelopathy, OPLL, cervical spine, degenerative cervical myelopathy, cohort study, anterior cervical spine, reoperation, readmission, complications

\section{INTRODUCTION}

Degenerative cervical myelopathy is one of the most common causes of spinal cord dysfunction, and it results from cervical spondylosis or ossification of the posterior longitudinal ligament, among other diagnoses occurring less frequently. ${ }^{1-3}$ Symptoms typically consist of upper and/or lower sensorimotor dysfunction. Surgical decompression of the spinal cord is effective in halting the disease progression and allowing for some neurologic recovery. ${ }^{1,2}$ Multilevel decompressions are commonly performed in a posterior fashion using laminectomy with fusion or laminoplasty. Motion preservation occurs with laminoplasty, while avoid- ing the well-known sequelae of laminectomy alone, which includes segmental instability, kyphotic deformity, perineural adhesions, and late neurologic deteriorations. ${ }^{4,5}$ Laminoplasty has proven to be a safe and effective procedure that also maintains cervical stability. ${ }^{4,6}$ In a recent systematic review and meta-analysis, clinical outcomes were similar when comparing laminoplasty to laminectomy with fusion, whereas some independent studies have demonstrated superior clinical outcomes and subjective improvement with decreased complications following laminoplasty when performed on a neutral or kyphotic cervical spine., ${ }^{4,7}$ Evidence suggests that motion is preserved. Machino et $\mathrm{al}^{5}$ reported that $87.9 \%$ of cervical range of motion was 
preserved. However, few studies have reported the reoperation rates following laminoplasty, and those reported have ranged from $0 \%$ to $13 \%{ }^{4,8,9}$ The purposes of this study were to determine: (1) the rate of acute postoperative complications leading to reoperations, (2) the late reoperation rate, and (3) the risk factors associated with conditions leading to reoperation.

\section{METHODS}

A retrospective review of all patients undergoing open-door cervical laminoplasty at our institution between January 1, 2005, and October 31, 2012, was performed. Inclusion criteria included minimum 2year follow-up, which consisted of either a clinic visit or telephone interview. Telephone interviews were attempted for patients with a final clinic visit less than 2 years after the laminoplasty procedure. Patients with prior anterior cervical procedures were not excluded, and those that had concomitant posterior procedures performed at the time of the laminoplasty were not excluded either. All surgeries were performed at 1 of 2 hospitals and performed by 7 fellowship-trained spine surgeons. The laminoplasty technique as described by Hirabayashi et al ${ }^{10}$ was performed on all patients.

Chart reviews were performed to collect the preoperative age; sex; body mass index; medical comorbidities, including diabetes and chronic obstructive pulmonary disease; prior cervical procedures; worker's compensation status; and duration of symptoms. Patient function was stratified according to the preoperative Ranawat classification. Operative records were reviewed to determine the cervical levels included in the laminoplasty procedure, estimated blood loss, and whether any concomitant procedures, such as laminectomy, arthrodesis, and/or foraminotomies, were performed. Postoperative notes were reviewed to determine whether any complications demanding reoperations occurred at any time following surgery as well as calculating time to reoperation. Reoperations were stratified as those occurring for acute postoperative complications, such as infection, wound-related issues, and malpositioned hardware, or those occurring for other reasons. The C5 palsy rate was also determined by review of postoperative clinic notes. For those patients with the last clinic visit occurring less than 2 years from the procedure, telephone interviews were attempted to determine whether additional cervical procedures were per-
Table 1. Demographics.

Male/female, $\mathrm{n}$

Median age, y (range)

Median BMI (range)

Median duration of symptoms, mo (range)

Median follow-up, y (range)

Diabetes, $\%$

COPD, \%

Worker's compensation status, \%

$132 / 90$ $56.2(30-86)$

$29.08(18.29-59.06)$

$8.5(1-288)$

$4.97(0-10)$

21

4

9.9

Abbreviations: BMI, body mass index; COPD, chronic obstructive pulmonary disease.

formed outside of our group and hospital system. A minimum of 3 attempts were made to contact patients via telephone before defining patients as lost to follow-up.

When available, preoperative imaging was reviewed. Radiographic measurements were obtained on plain films. The measurements included the $\mathrm{C} 2$ to C7 alignment and the Pavlov ratio at the sixth cervical vertebral level. Measurements on preoperative advanced imaging included the presence or absence of increased T2 cord signal and the sagittal canal diameter at the sixth cervical vertebral level.

\section{Statistical Analysis}

A number of variables were collapsed to define dichotomous variables for statistical analysis: body mass index threshold of 30; symptoms duration longer than 9 months; Ranawat class of I/II and IIIa/IIIb; stenosis $<0.8$; and sagittal canal $<12$. Standard descriptive statistics were calculated. For categoric variables, $\chi^{2}$ tests and Fisher exact tests were used to determine differences in proportions. Relative risks and 95\% confidence intervals were also calculated. An a priori level of significance of .05 was used for all tests.

\section{Study Sample}

A total of 266 patients underwent open-door cervical laminoplasty between January 1, 2005, and October 31,2012 . A total of 222 patients $(83 \%)$ had a minimum 2-year follow up consisting of either a clinic visit or a telephone interview and were included in this cohort. The demographics details of the study sample are presented in Table 1. There were 132 men and 90 women. The mean age was 56.2 years (range, 30-86 years). The median duration of symptoms was 8.5 months (range, 1288 months). Most patients were Ranawat class II $(33 \%)$ or IIIA $(48 \%)$ (Table 2$)$. The average followup was 4.97 years, with a range of 0 to 10 . One patient who required a reoperation died for 
Table 2. Ranawat class.

\begin{tabular}{lc}
\hline Ranawat Classification & No. of Patients \\
\hline Class I & 38 \\
Class II & 74 \\
Class IIIA & 106 \\
Class IIIB & 4 \\
\hline
\end{tabular}

unrelated reasons prior to the 2-year follow up. The reason for surgery was cervical spondylotic myelopathy (CSM) in 180 patients $(82 \%)$, ossification of the posterior longitudinal ligament (OPLL) in 21 patients $(9 \%)$, and stenosis in 21 patients $(9 \%)$.

\section{RESULTS}

\section{Surgery-Related Results}

The median number of laminoplasty levels performed was 4 (interquartile range, 3-5), and the frequency of each level is demonstrated in Figure 1. A total of 111 patients $(50 \%)$ had concomitant laminectomies, $6(3 \%)$ had arthrodesis and 84 (38\%) had concomitant foraminotomies performed as further detailed in Figures 2 to 4 . The median estimated blood loss was $250 \mathrm{~mL}$.

\section{Radiographic Evaluation}

A complete preoperative radiographic evaluation was available for 138 of the 222-patient cohort $(62.1 \%)$. A complete preoperative workup consisted of both preoperative plain films and magnetic resonance imaging or computed tomography myelogram. The median preoperative sagittal alignment from $\mathrm{C} 2$ to $\mathrm{C} 7$ was $7^{\circ}$ of lordosis, with a range from $38^{\circ}$ of lordosis to $6^{\circ}$ of kyphosis. The mean C6 Pavlov ratio was 0.8 , with a range from 0.5 to 1.59 . A total of 71 of the 170 patients with available magnetic resonance images had increased $\mathrm{T} 2$ cord signal change. The median C6 sagittal canal

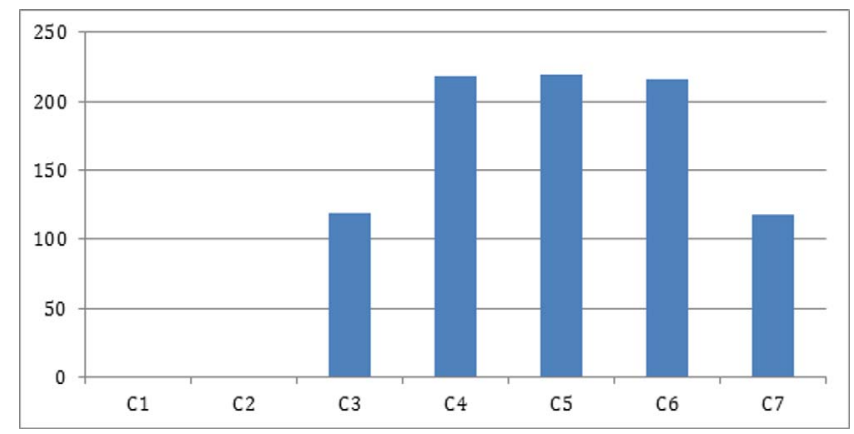

Figure 1. Frequency of laminoplasty surgical levels. The levels are not isolated, and many will be on the same patient.

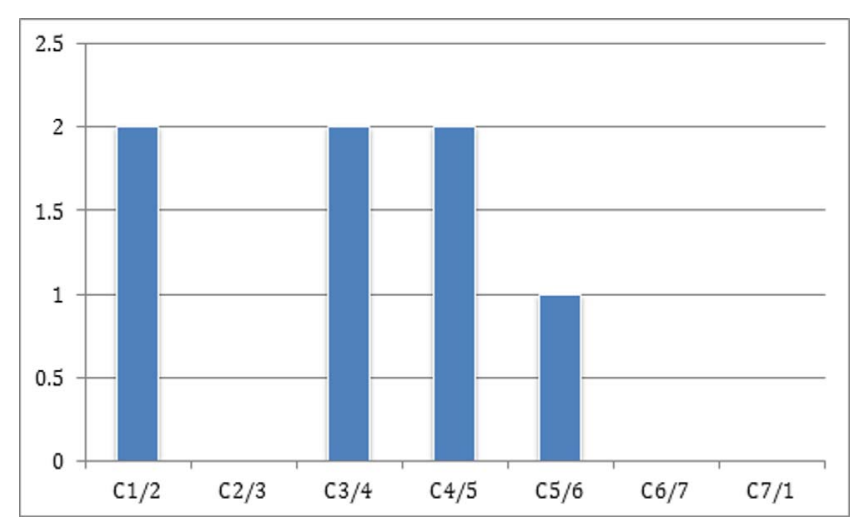

Figure 2. Frequency of fusion surgical levels. The levels are not isolated, and many will be on the same patient.

diameter was $9.4 \mathrm{~mm}$, with a range from 3.1 to $13.6 \mathrm{~mm}$. These results are demonstrated in Table 3.

\section{Reoperations and C5 Palsy Rate}

A total of 26 patients underwent 30 reoperations $(13.5 \%)$ out of the 222-patient cohort. A total of 15 patients had 16 reoperations $(7.2 \%)$ in the acute postoperative period: 10 patients $(4.5 \%)$ for infection receiving at least 1 irrigation and debridement, 3 patients $(1.3 \%)$ for hardware-related issues, and 3 patients $(1.3 \%)$ for posterior cervical wound issues, 1 of which was for a cerebrospinal fluid fistula. A total of 13 patients underwent 14 reoperations $(6.3 \%)$ outside of the acute postoperative period: 6 patients $(2.7 \%)$ for the development of a new radiculopathy, 3 patients $(1.3 \%)$ for recurrent myelopathy, 2 patients $(0.90 \%)$ for the development of neurologic symptoms with a kyphotic deformity, and 1 patient $(0.45 \%)$ who fell and struck his head, resulting in a posttraumatic focal kyphotic deformity successfully treated with a C5 to C7 anterior cervical discectomy and fusion. Three patients

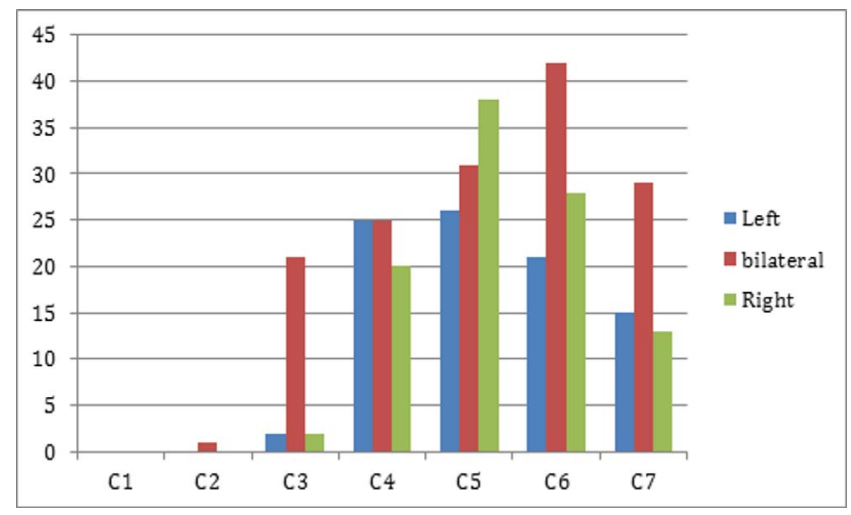

Figure 3. Frequency of foraminotomy surgical levels. The levels are not isolated, and many will be on the same patient. 


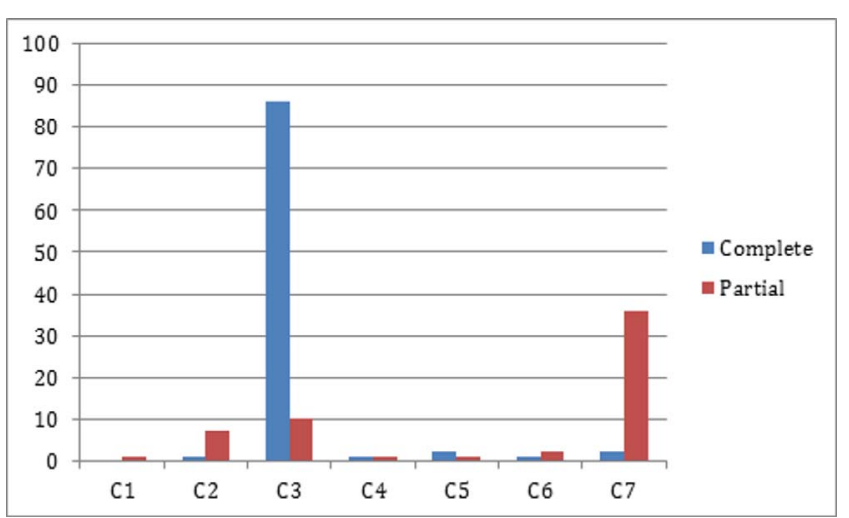

Figure 4. Frequency of laminectomy surgical levels. The levels are not isolated, and many will be on the same patient.

reported additional procedures performed outside our hospital network: One patient underwent a reoperation for a new radiculopathy, which was included in the numbers above. A total of 2 of the 3 patient records were unable to be obtained and reviewed. We had an 18 of $222(8.1 \%)$ C5 palsy rate. A patient was included if $\mathrm{C} 5$ palsy was mentioned at any point in the clinic notes, regardless of the severity.

Overall, patients who had a concomitant partial or complete laminectomy demonstrated a significantly $(P=.03)$ higher reoperation rate compared with those who did not. This factor remained significant when comparing only those patients in the late reoperation cohort $(P=.008)$. No other statistically significant associations were found.

\section{DISCUSSION}

This study demonstrated a $13.5 \%$ reoperation rate after open-door cervical laminoplasty, with an average follow-up of nearly 5 years. This is consistent with previously reported rates. Highsmith et $\mathrm{al}^{8}$ retrospectively reviewed 56 patients, 30 of whom underwent laminoplasty and 26 of whom underwent laminectomy and fusion. The follow-ups averaged 42.3 months and 41.3 months, respective1y. ${ }^{8}$ They demonstrated a $13 \%$ reoperation rate in the laminoplasty group compared with a $27 \%$ reoperation rate in the laminectomy and fusion group. In both cohorts, reported reoperations were for perioperative complications, such as wound infection or hardware malposition, except for 1 patient in the laminectomy and fusion group who required an anterior discectomy and fusion for an inadequate decompression for residual radiculopathy. ${ }^{8}$ Heller et $\mathrm{al}^{4}$ performed a matched cohort
Table 3. Radiographic evaluation.
C2-C7 Angle

Torg ratio (C6)

Increased $\mathrm{T} 2$ cord signal

C6 sagittal canal diameter, $\mathrm{mm}$ $7^{\circ}$ Lordosis $\left(38^{\circ}\right.$ Lordosis

to $6^{\circ}$ Kyphosis)

$0.8(0.5-1.59)$

71 patients

$9.4(3.1-13.6)$ retrospective analysis of 13 laminoplasty patients and 13 laminectomy and fusion patients and reported a $0 \%$ reoperation rate in the laminoplasty cohort, compared with a $15 \%$ reoperation rate in the laminectomy and fusion group. In that study, mean follow-up was 25.5 months for the laminectomy with fusion cohort and 26.2 months for the laminoplasty cohort. Reasons for reoperations in the laminectomy and fusion cohort include adjacent degeneration and deep infection. Other reported complications that did not require reoperations include myelopathy progression, pseudarthrosis, broken hardware, kyphotic deformity, and graft site pain, compared with no complications in the laminoplasty cohort. However, Heller et $\mathrm{al}^{4}$ did discuss an overall institution complication rate of $8 \%$ for laminoplasty. Woods et $\mathrm{al}^{9}$ retrospectively reviewed 121 patients with multilevel CSM or myeloradiculopathy with a minimum 6.7-month follow-up and an average 24-month follow-up: 39 patients underwent laminoplasty and 82 underwent laminectomy and fusion. ${ }^{9}$ The laminoplasty group had 2 reoperations for a $5.1 \%$ reoperation rate: one for recurrent stenosis due to a large anterior osteophyte and the other for persistent and progressive radiculopathy postoperatively. The laminectomy and fusion group underwent 3 reoperations for a $3.6 \%$ reoperation rate: 2 patients led to formal revisions and 1 for persistent posterior wound drainage and subsequent dehiscence. ${ }^{9}$ Yang et $\mathrm{al}^{11}$ retrospectively reviewed 141 patients who underwent either laminoplasty or laminectomy and fusion for CSM and reported zero reoperations in the laminectomy and fusion cohort and 1 reoperation in the laminoplasty cohort, for a laminoplasty reoperation rate of $1.3 \%$. Interestingly, this was performed for restenosis 3 years after the patient's laminoplasty and reportedly for natural progression of degeneration. To our knowledge, few previous studies have reported reoperations for the development of new neurologic symptoms. We found that $6.3 \%$ of our reoperations occurred for the development of new neurologic symptoms, and, on average, 2 years following the index procedure. Of these, 3 were 
performed for focal pathology below the index procedure, 7 for focal pathology within the levels of the index procedure, 3 conversions to posterior cervical fusions, and 1 unknown. Machino et $\mathrm{al}^{5}$ reported $87.9 \%$ preserved motion with laminoplasty, and this motion preservation could allow for the development of new neurologic symptoms within the levels of the index procedure.

Laminectomy without fusion, although now rarely performed, is associated with a high incidence of postlaminectomy cervical kyphotic deformity, with Kaptain et $\mathrm{al}^{12}$ reporting a $21 \%$ incidence. Laminoplasty has also been associated with some reports of postoperative kyphotic sagittal alignment, and Suk et $\mathrm{al}^{13}$ reported a $10.6 \%$ incidence of postoperative kyphosis, with a mean kyphosis angle of $12.2^{\circ}$ in those patients. They also identified 3 preoperative factors that led to an increased risk of postoperative kyphosis in their cohort: a preoperative diagnosis of myelopathy, preoperative lordosis angle $<10^{\circ}$ in the neutral position, and a preoperative kyphotic angle during flexion that is greater than a lordotic angle during extension. ${ }^{13}$ Two known patients in our cohort ultimately needed a reoperation for postoperative kyphotic alignment following laminoplasty.

Shou et $\mathrm{al}^{14}$ conducted a meta-analysis based on 13621 patients in a combined 79 studies and showed an overall C5 palsy incidence of $5.3 \%$. This meta-analysis included anterior and posterior decompressive procedures. Laminoplasty alone demonstrated a $5.1 \%$ pooled incidence when combining 4986 patients in 39 studies. ${ }^{14}$ This is consistent with a Sakaura et al ${ }^{15}$ review that demonstrated a $4.6 \%$ average reported incidence, with other reported rates ranging from 0 to $30 \%$. Chiba et al ${ }^{16}$ reported a $10 \%$ segmental motor paralysis occurring in either C5 or C6, and all completely resolved by 2 years. Our study demonstrated an $8.1 \%$ incidence.

Our study is not without limitations. First, inherent retrospective limitations are present. Many patients had also been given "as needed" follow-up prior to the 2-year postoperative period. However, through repeated telephone calls, we were able to obtain an $83 \%$ follow-up. Also, several patients had reoperations at other hospitals, and we were not able to obtain outside medical records for all patients, even though multiple attempts were made. Some patients were able to provide some information over the phone, including dates of procedures. And, although our purpose and intent was to determine the reoperation rate, postoperative clinical scores and outcomes were not obtained and our preoperative radiographic parameters were limited and without any postoperative evaluation.

\section{CONCLUSION}

Our cohort had an overall $13.5 \%$ reoperation rate, with a $6 \%$ reoperation rate outside of the acute postoperative period related to the development of new neurologic symptoms. Although the preserved motion following laminoplasty may allow for the development of new neurologic symptoms within previously operative levels, the reoperation rate continues to compare favorably with reported rates of laminectomy and fusion and remains a reasonable option for the surgical management of multilevel degenerative cervical myelopathy.

\section{REFERENCES}

1. Emery SE. Cervical spondylotic myelopathy: diagnosis and treatment. J Am Acad Orthop Surg. 2001;9(6):376-388.

2. Lawrence BD, Jacobs WB, Norvell DC, Hermsmeyer JT, Chapman JR, Brodke DS. Anterior versus posterior approach for treatment of cervical spondylotic myelopathy: a systematic review. Spine (Phila Pa 1976). 2013;38(22 suppl 1):S173-S182.

3. Wilson JR, Barry S, Fischer DJ, et al. Frequency, timing, and predictors of neurological dysfunction in the nonmyelopathic patient with cervical spinal cord compression, canal stenosis, and/or ossification of the posterior longitudinal ligament. Spine (Phila Pa 1976). 2013;38(22 suppl 1):S37-S54.

4. Heller JG, Edwards CC 2nd, Murakami H, Rodts GE. Laminoplasty versus laminectomy and fusion for multilevel cervical myelopathy: an independent matched cohort analysis Spine (Phila Pa 1976). 2001;26(12):1330-1336.

5. Machino M, Yukawa Y, Hida T, et al. Cervical alignment and range of motion after laminoplasty: radiographical data from more than 500 cases with cervical spondylotic myelopathy and a review of the literature. Spine (Phila Pa 1976). 2012;37(20):E1243-E1250.

6. Nowinski GP, Visarius H, Nolte LP, Herkowitz HN. A biomechanical comparison of cervical laminaplasty and cervical laminectomy with progressive facetectomy. Spine (Phila $\mathrm{Pa}$ 1976). 1993;18(14):1995-2004.

7. Phan K, Scherman DB, Xu J, Leung V, Virk S, Mobbs RJ. Laminectomy and fusion vs laminoplasty for multi-level cervical myelopathy: a systematic review and meta-analysis. Eur Spine J. 2017;26(1):94-103.

8. Highsmith JM, Dhall SS, Haid RW Jr, Rodts GE Jr, Mummaneni PV. Treatment of cervical stenotic myelopathy: a cost and outcome comparison of laminoplasty versus laminectomy and lateral mass fusion. $J$ Neurosurg Spine. 2011;14(5):619-625.

9. Woods BI, Hohl J, Lee J, Donaldson W 3rd, Kang J. Laminoplasty versus laminectomy and fusion for multilevel 
cervical spondylotic myelopathy. Clin Orthop Relat Res. 2011;469(3):688-695.

10. Hirabayashi K, Watanabe K, Wakano K, Suzuki N, Satomi K, Ishii Y. Expansive open-door laminoplasty for cervical spinal stenotic myelopathy. Spine (Phila Pa 1976). 1983;8(7):693-699.

11. Yang L, Gu Y, Shi J, et al. Modified plate-only opendoor laminoplasty versus laminectomy and fusion for the treatment of cervical stenotic myelopathy. Orthopedics. 2013;36(1):e79-e87.

12. Kaptain GJ, Simmons NE, Replogle RE, Pobereskin L. Incidence and outcome of kyphotic deformity following laminectomy for cervical spondylotic myelopathy. J Neurosurg. 2000;93(2 suppl):199-204.

13. Suk KS, Kim KT, Lee JH, Lee SH, Lim YJ, Kim JS. Sagittal alignment of the cervical spine after the laminoplasty. Spine (Phila Pa 1976). 2007;32(23):E656-E660.

14. Shou F, Li Z, Wang H, Yan C, Liu Q, Xiao C. Prevalence of c5 nerve root palsy after cervical decompressive surgery: a meta-analysis. Eur Spine J. 2015;24(12):2724-2734.

15. Sakaura H, Hosono N, Mukai Y, Ishii T, Yoshikawa H. C5 palsy after decompression surgery for cervical myelopathy: review of the literature. Spine (Phila Pa 1976). 2003;28(21):24472451.
16. Chiba K, Ogawa Y, Ishii K, et al. Long-term results of expansive open-door laminoplasty for cervical myelopathyaverage 14-year follow-up study. Spine (Phila Pa 1976). 2006;31(26):2998-3005.

Disclosures and COI: The authors received no funding for this study and report no conflicts of interest.

Corresponding Author: Susan M. Odum, PhD, OrthoCarolina Research Institute Inc., 2001 Vail Ave, Ste 300, Charlotte, NC 28207. Phone: (704) 323-2265; Fax: (704) 323-3967; Email: susan. odum@orthocarolina.com.

Published 21 December 2018

This manuscript is generously published free of charge by ISASS, the International Society for the Advancement of Spine Surgery. Copyright (c) 2018 ISASS. To see more or order reprints or permissions, see http://ijssurgery.com. 